\title{
Protein NLRC5
}

National Cancer Institute

\section{Source}

National Cancer Institute. Protein NLRC5. NCI Thesaurus. Code C132033.

Protein NLRC5 (1866 aa, 205 kDa) is encoded by the human NLRC5 gene. This protein is involved in signaling pathways and antiviral responses. 